\title{
A new algorithm for solving the nonlinear Lane-Emden equations arising in astrophysics
}

\author{
Mohamed R. Ali ${ }^{1}$ (D) \\ (C) Springer Nature Switzerland AG 2019
}

\begin{abstract}
Hybrid orthonormal Bernstein and block-pulse function wavelet method for many phenomena in mathematical physics and astrophysics is considered. We use a fundamental administrator and convert Lane-Emden conditions into necessary conditions. The upsides of utilizing the proposed strategy are introduced. At that point, an effective error estimation for the proposed technique is additionally presented lastly a few examinations and their numerical arrangements are given; and looking at between the numerical outcomes acquired from alternate strategies, we demonstrate the high exactness and productivity of the proposed strategy. Our method is characterized then the singular equations are transformed to Volterra integro-differential equations. We modify this equations to an algebraic system of equations. The solution to this application is achieved by solving this system and the constructed solutions are on approximation form. The acquired results guarantee the method provides a truncate solution to the Lane-Emden equations.
\end{abstract}

Keywords Hybrid orthonormal Bernstein and block-pulse functions - Wavelet method · Convergence analysis - LaneEmden equation · Gaussian integration · Collocation method

\section{Introduction}

Many practical problems arising in numerous branches of science and ocean engineering require solving boundary value problems. Different Lane-Emden type conditions are known to happen in nonlinear beginning quality issues which have a peculiarity at the beginning. The logical arrangements of these sorts of conditions are constantly conceivable in the area of the point $x=0$. These conditions moreover portray numerous marvels of material science and astronomy, for example, those including certain parts of an outstanding structure, warm history of an around billow of gas; geothermal gas circles and thermionic flows. HOBW has been used for obtaining numerical spectral solutions for handling some of these kinds of Ocean Engineering problems. The LEE has fundamental importance in the field of radioactive cooling, modeling of clusters of galaxies, Physics, astrophysics, and nonlinear mechanics are used to depict several equations [1-7]. It has also been proven to be more versatile in the examination of a variety of situations which include the analysis of isothermal cores, convective stellar interiors and fully degenerate stellar configurations and formulated as:

$y^{\prime \prime}(x)+\frac{k}{x} y^{\prime}(x)+f(x, y)=h(x), \quad x, k \geq 1$

with initial conditions (IC) as:

$y(0)=y_{0}, \quad y^{\prime}(0)=y_{1}$,

where $y_{0}, y_{1}$ are constants, $h(x), f(x, y)$ are continuous functions.ff we take $f(x, y)=f(y)=y^{n}$ then Eq. (1) is given by:

$y^{\prime}(x)+\frac{k}{x} y^{\prime}(x)+f(y)=h(x)$

with Dirichlet, Neumann, and Neumann-Robin boundary conditions

Mohamed R. Ali, mohamed.reda@bhit.bu.edu.eg| 'Department of Mathematics, Faculty of Engineering, Benha University, Benha, Egypt. 
$y(0)=y_{0}, \quad y(1)=y_{1}$,

$y^{\prime}(0)=c_{0}, \quad y^{\prime}(1)=c_{1}$,

$y^{\prime}(0)=0, \quad v_{0} y(1)+v_{2} y^{\prime}(0)=v_{3}$,

which models most phenomena in mathematics for the values of $f(y)$.

For $y_{0}=1$, this equation is the LEE.

The name of this equation is due to the two astrophysicists Jonathan Lane and Robert Emden [1]. Equation (1) has a singularity at $x=0$. An effective strategy was established in [5] to modify the Lane-Emden to equivalent Volterra integral equations (VIE) off any order. The VIE in [8], combined with (ADM), to address the singularity issue. This strategy was proven to be reliable and efficient as confirmed in [9]. The physical structure introduced in [10] the mother wavelet. In which parameter of dilation $a$ and parameter of a translation $b$ vary continuously.

$\psi_{a, b}(t)=|a|^{\frac{-1}{2}} \psi\left(\frac{t-b}{a}\right), \quad a, b \in \mathrm{R}, a \neq 0$

By letting $a$ and $b$ be discrete values such as $a=a_{0}^{-k}$, $b=n b_{0} a_{0}^{-k}, a_{0}>1, b_{0}>0$, where $n$ and $k$ are positive integers, we attain the family of discrete wavelets:

$\psi_{k, n}(t)=\left|a_{0}\right|^{\frac{k}{2}} \psi\left(a_{0}^{k} t-n b_{0}\right), \quad n, k \in Z^{+}$

Then we see that $\psi_{k, n}(t)$ forms a wavelet basis for $L^{2}(R)$. In particular, when, $a_{0}=2, b_{0}=1$, then $\psi_{k, n}(t)$ forms a basis. Here, $\operatorname{HOBW} W_{i, j}(t)=\operatorname{HOBW}(k, i, j, t)$ involves four arguments, $i=1, \ldots, 2^{k-1}, k$ is to be any positive integer, $j$ is the degree of the Bernstein polynomials, and $t$ is the normalized time. They $\mathrm{HOBW}_{i, j}(t)$ are defined on $[0,1)$ as $[11,12]$ :

$\operatorname{HOBW}_{i, j}(t)= \begin{cases}2^{\frac{k-1}{2}}\left(\begin{array}{c}n \\ j\end{array}\right)\left(2^{k-1} t-i+1\right)^{j}\left(1-\left(2^{k-1} t-i+1\right)\right)^{n-j} & \frac{i-1}{2^{k-1}} \leq t<\frac{i}{2^{k-1}} \\ 0 & \text { otherwise }\end{cases}$

Many researchers focused to give a truncate solution to these equations and many methods were proposed. A general study has been given in [10] to construct both correct and series solutions to Lane-Emden equations through ADM. In [11-18] introduced Bernstein, several methods for numerical solutions of VIDE form the unique Emden-Fowler initial value problems. In [19] approach developed to obtain analytical-numerical solutions to two separate Lane-Emden problems. In [20-24] introduced the exponential transformation to resolve the difficulty of a singular point to solve the LEE.

The paper introduces as. In Sect. 2, we present the properties of the HOBW and the approximation of the function using it. In Sect. 3, We establish that the VIDE is the singular Lane-Emden equation. In Sect. 4, we use HOBW method and its convergence analysis for getting the solution VIDE form of the singular Lane-Emden equation. The efficiency of our method is characterized by solving numerical examples in Sect. 5. Conclusion part is given in Sect. 6 .

\section{The HOBW method and the operational matrix of the integration}

\subsection{Wavelets and the HOBW method}

Wavelets constitute a group of functions constructed from dilation and translation of a single function $\psi(x)$ called where $i=1,2, \ldots ., 2^{k-1}, j=0,1, \ldots, M-1$ and $k$ is a positive integer. Thus, we attain our new basis as $\left\{\mathrm{HOBW}_{1,0}, \mathrm{HOBW}_{1,1}, \ldots, \mathrm{HOBW}_{2^{k-1}, M-1}\right\}$ and any function is truncated with them.

The HOBW detect orthonormal basis is:

$\left(H O B W_{i j}(t), H O B W_{i^{\prime} j^{\prime}}(t)\right)= \begin{cases}1 & (i, j)=\left(i^{\prime}, j^{\prime}\right) \\ 0 & (i, j) \neq\left(i^{\prime}, j^{\prime}\right)\end{cases}$

where $(.,$.$) called the inner product in L^{2}[0,1)$. The HOBW has compact support $\left[\frac{i-1}{2^{k-1}}, \frac{i}{2^{k-1}}\right], i=1, \ldots, 2^{k-1}$.

\subsection{Function approximation by the HOBW functions}

Any function $y(t)[10-12]$, which is integrable in $[0,1)$, is truncated by the HOBW method as follows:

$$
\begin{aligned}
y(t) & =\sum_{i=1}^{\infty} \sum_{j=0}^{\infty} c_{i j} \operatorname{HOBW}_{i j}(t), i=1,2, \ldots, \infty, \\
j & =0,1,2, \ldots, \infty, t \in[0,1)
\end{aligned}
$$

where the HOBW coefficients $c_{i j}$ calculated as given below:

$$
c_{i j}=\frac{\left(y(t), \operatorname{HOBW}_{i j}(t)\right)}{\left(\operatorname{HOBW}_{i j}(t), \operatorname{HOBW}_{i j}(t)\right)}
$$

We prune $y(t)$ by a series as follows: 
$y(t)=\sum_{i=1}^{2^{k-1}} \sum_{j=0}^{M-1} c_{i j} H O B W_{i j}(t)=C^{T} \operatorname{HOBW}(t)$

where $\operatorname{HOBW}(t)$ and $C$ are $\left(2^{k-1}\right)(M-1) \times 1$ vectors given by

$$
\begin{aligned}
\operatorname{HOBW}(t)= & {\left[\mathrm{HOBW}_{10}, \mathrm{HOBW}_{11}, \ldots, \mathrm{HOBW}_{1(M-1)},\right.} \\
& \mathrm{HOBW}_{20}, \mathrm{HOBW}_{21}, \ldots, \mathrm{HOBW}_{2(M-1)}, \ldots \ldots, \\
& \left.\left.\mathrm{HOBW}_{2^{k-1} 0}, \ldots, \mathrm{HOBW}_{2^{k-1}(M-1)}\right]\right]^{T}
\end{aligned}
$$

and

$$
\begin{aligned}
C= & {\left[c_{10}, c_{11}, \ldots, c_{1(M-1}\right), c_{20}, c_{21}, \ldots, } \\
& \left.C_{2(M-1)}, \ldots, C_{2^{k-1} 0}, \ldots, C_{2^{k-1}(M-1)}\right] .^{T}
\end{aligned}
$$

$C=D^{-1}<y(t), \operatorname{HOBW}(t)>$,

where

$$
\begin{aligned}
& D=<\operatorname{HOBW}(t), \operatorname{HOBW}(t)>\text {, } \\
& =\int_{0}^{1} \operatorname{HOBW}(t) \cdot \operatorname{HOBW}^{T}(t) d t \\
& =\left(\begin{array}{cccc}
D_{1} & 0 & \cdots & 0 \\
0 & D_{2} & \cdots & 0 \\
\vdots & & \ddots & 0 \\
0 & 0 & \cdots & D_{M}
\end{array}\right)
\end{aligned}
$$

Then, $D_{i}\left(i=1,2, \ldots, 2^{k-1}\right)$ is defined as follows:

$$
\begin{aligned}
\left(D_{n}\right)_{i+1, j+1} & =\int_{\frac{i-1}{2^{k-1}}}^{\frac{i}{2^{k-1}}} \operatorname{HOBW}_{i, n}\left(2^{k-1} t-i+1\right) \operatorname{HOBW}_{j, n}\left(2^{k-1} t-i+1\right) d t \\
\left(D_{n}\right)_{i+1, j+1} & =\frac{1}{2^{k-1}} \int_{0}^{1} \operatorname{HOBW}_{i, n}(t) \operatorname{HOBW}_{j, n}(t) d t \\
\left(D_{n}\right)_{i+1, j+1} & =\frac{\left(\begin{array}{c}
n \\
i
\end{array}\right)\left(\begin{array}{c}
n \\
j
\end{array}\right)}{2^{k-1}(2 n+1)\left(\begin{array}{c}
2 n \\
i+j
\end{array}\right)}
\end{aligned}
$$

We can also truncate the function $k(x, t) \in L[0,1]$ as follows:

$k(x, t) \approx \operatorname{HOBW}^{T}(x) K \operatorname{HOBW}(t)$,

where $K$ is a $\left(2^{k-1}\right)(M-1) \times\left(2^{k-1}\right)(M-1)$ matrix that we attain as:

$K=D^{-1}<\operatorname{HOBW}(x)<k(x, t), \operatorname{HOBW}(t)>>D^{-1}$

\subsection{Multiplication of the hybrid functions}

We can evaluateHOBW ${ }_{\left(\left(2^{k-1}\right)(M-1) \times 1\right)}(t) \mathrm{HOBW}_{\left(\left(2^{k-1}\right)(M-1) \times 1\right)}^{T}(t)$ for VIE of the second kind via the HOBW functions as detailed below.

Let the product of $\operatorname{HOBW}_{\left(\left(2^{k-1}\right)(M-1) \times 1\right)}(t)$ and $\operatorname{HOBW}_{\left(\left(2^{k-1}\right)(M-1) \times 1\right)}^{T}(t)$ be given by

$$
\begin{aligned}
& \operatorname{HOBW}_{\left(\left(2^{k-1}\right)(M-1) \times 1\right)}(t) \operatorname{HOBW}_{\left(\left(2^{k-1}\right)(M-1) \times 1\right)}^{T}(t) \\
& \cong M_{\left(\left(2^{k-1}\right)(M-1) \times\left(2^{k-1}\right)(M-1)\right)}(t)
\end{aligned}
$$

where

With the recursive formulas, we calculate $M_{\left(\left(2^{k-1}\right)(M-1) \times\left(2^{k-1}\right)(M-1)\right)}(t)$ for any $k$ and $M$.

The matrix $M_{\left(\left(2^{k-1}\right)(M-1) \times\left(2^{k-1}\right)(M-1)\right)}(t)$ satisfies the relation: 


$$
\begin{aligned}
& M_{\left(2^{k-1}(M-1) \times 2^{k-1}(M-1)\right)}(t) C_{\left(2^{k-1}(M-1) \times 1\right)} \\
& =\tilde{C}_{\left(2^{k-1}(M-1) \times 2^{k-1}(M-1)\right)} \operatorname{HOBW}_{\left(2^{k-1}(M-1) \times 1\right)}(t) \quad y^{\prime}(x)=\int_{0}^{x}\left(\frac{t^{k}}{x^{k}}\right) h(t) d t-\int_{0}^{x}\left(\frac{t^{k}}{x^{k}}\right) f(y(t)) d t,
\end{aligned}
$$

where $\tilde{C}_{\left(2^{k-1}(M-1) \times 2^{k-1}(M-1)\right)}$ is the matrix coefficient. We con-

sider the case when $k=3$ and $M=4$. Thus, we have

$M_{(16) \times 16)}(t)=M_{(16) \times 16)}(t)=\left[\begin{array}{llll}\operatorname{HOBW}_{10}(t) \operatorname{HOBW}_{10}(t) & \operatorname{HOBW}_{10}(t) \mathrm{HOBH}_{20}(t) & \cdots & \operatorname{HOBW}_{10}(t) \operatorname{HOBW}_{43}(t) \\ \operatorname{HOBW}_{20}(t) \operatorname{HOBW}_{10}(t) & \operatorname{HOBW}_{20}(t) \mathrm{HOBH}_{20}(t) & \cdots & \operatorname{HOBW}_{20}(t) \operatorname{HOBW}_{43}(t) \\ \operatorname{HOBW}_{30}(t) \operatorname{HOBW}_{10}(t) & \operatorname{HOBW}_{30}(t) \mathrm{HOBH}_{20}(t) & \cdots & \operatorname{HOBW}_{30}(t) \operatorname{HOBW}_{43}(t) \\ \vdots & \vdots & \cdots & \vdots \\ \operatorname{HOBW}_{43}(t) \operatorname{HOBW}_{10}(t) & \operatorname{HOBW}_{43}(t) \operatorname{OBH}_{20}(t) & \cdots & \operatorname{HOBW}_{43}(t) \operatorname{HOBW}_{43}(t)\end{array}\right]$

The coefficient matrix $\tilde{C}_{\left(2^{k-1} M-1 \times 2^{k-1} M-1\right)}$ is determined by

$\tilde{C}_{\left(2^{k-1} M \times 2^{k-1} M\right)}=\left[\begin{array}{cccc}C_{0} & 0 & 0 & 0 \\ 0 & C_{1} & 0 & 0 \\ 0 & 0 & C_{2} & 0 \\ 0 & 0 & 0 & C_{3}\end{array}\right]$

where $C_{i}, i=0,1,2,3$ are $4 \times 4$ matrices.

Several of the integral $\int_{a(x)}^{b(x)} F(x, t) d t$ using Leibniz rule as:

$\frac{d}{d x} \int_{a(x)}^{b(x)} F(x, t) d t=F(x, b(x)) \frac{d b}{d x}-F(x, a(x)) \frac{d a}{d x}+\int_{a(x)}^{b(x)} \frac{d F}{d x} d t$

Where $F(x, t)$ and $\frac{d F}{d x}$ are continuous in the domain $D$ in the $x t$ - plane that contains the region $R, a \leq x \leq \beta, t_{0} \leq t \leq t_{n}$ and $a(x), b(x)$ are defined functions having continuous derivatives for $a \leq x \leq \beta$. A global Leibniz rule presented modifies VIE to several equations.

\section{VIDE form the Lane-Emden type}

In this section, we discuss the LEE of the shape factor of the form

$y^{\prime \prime}(x)+\frac{k}{x} y^{\prime}(x)+f(y)=h(x), \quad y(0)=\alpha, \quad y^{\prime}(0)=0, \quad k>1$

where $f(y)$ can take any linear or nonlinear forms.

First, we set

$y(x)=\alpha-\frac{1}{k-1} \int_{0}^{x}\left(t\left(1-\frac{t^{k-1}}{x^{k-1}}\right) f(y(t))-h(t)\right) d t$,

to modify (18) to an integral form then differentiating Eq. (19) twice and using the Leibniz rule, we have

SN Applied Sciences

$$
\begin{aligned}
y^{\prime \prime}(x)= & h(x)-\int_{0}^{x} k\left(\frac{t^{k}}{x^{k+1}}\right) h((t)) d t-f(y(x)) \\
& +\int_{0}^{x} k\left(\frac{t^{k}}{x^{k+1}}\right) f(y(t)) d t
\end{aligned}
$$

If we multiply $y^{\prime}(x)$ in (20) by $\frac{k}{x}$ and add to $y^{\prime \prime}(x)$ in (21) we get Eq. (18). That is the VIDE is the LEE (18) that given by:

$$
\begin{gathered}
y^{\prime}(x)=\int_{0}^{x}\left(\frac{t^{k}}{x^{k}}\right) h(t) d t-\int_{0}^{x}\left(\frac{t^{k}}{x^{k}}\right) f(y(t)) d t, \\
k>1, \quad y(0)=\alpha .
\end{gathered}
$$

The VIE (10)-(11) were proved to be equivalent to the homogenous LEE (18).

For $k \rightarrow 1$, the integral form of Eq. (19) is

$y(x)=\alpha+\int_{0}^{x} t\left(\ln \left(\frac{t}{x}\right) f(y(t))-h(t)\right) d t$.

Based on this, we set $t$ the Lane-Emden equations in Volterra integral forms are as:

$\left\{\begin{array}{l}y(x)=\alpha+\int_{0}^{x} t\left(\ln \left(\frac{t}{x}\right) f(y(t))-h(t)\right) d t, \quad \text { for } k=1, \\ y(x)=\alpha-\frac{1}{k-1} \int_{0}^{x}\left(t\left(1-\frac{t^{k-1}}{x^{k-1}}\right) f(y(t))-h(t)\right) d t, \text { for } k>1 .\end{array}\right.$

\section{Application of HOBW method for Lane- Emden in its integrodifferential form}

Consider Eq. (22) acquired from Eq. (18). The unknown function $\mathrm{y}(\mathrm{x})$ is approximated by HOBW as 
$y(x) \cong \sum_{i=1}^{M} \sum_{j=0}^{n} c_{i j} \operatorname{HOBW}_{i j}(x)=C^{T} \operatorname{HOBW}(x)$.

First, integrating Eq. (22) and from the condition $y(0)=a$, one gets

$$
\begin{aligned}
y(x)= & \alpha+\int_{0}^{x}\left[\int_{0}^{z}\left(\frac{x^{k}}{z^{k}}\right) h(x) d x\right] d z \\
& -\int_{0}^{x}\left[\int_{0}^{z}\left(\frac{t^{k}}{z^{k}}\right) f(y(t)) d t\right] d z, \quad k \geq 1 .
\end{aligned}
$$

Then from Eqs. (23) and (24), we have

$$
\begin{aligned}
C^{T} \operatorname{HOBW}(x)= & \alpha+\int_{0}^{x}\left[\int_{0}^{z}\left(\frac{x^{k}}{z^{k}}\right) h(x) d x\right] d z \\
& -\int_{0}^{x}\left[\int_{0}^{z}\left(\frac{t^{k}}{z^{k}}\right) f(y(t)) d t\right] d z, \quad k \geq 1 .
\end{aligned}
$$

$=\alpha+\int_{0}^{x} H_{1}(z) d z-\int_{0}^{x} H_{2}(z) d z$

where,

$H_{1}(z)=\int_{0}^{z}\left(\frac{x^{k}}{z^{k}}\right) h(x) d x$,

where $D$ is a $\left(2^{k-1}\right)(M-1) \times\left(2^{k-1}\right)(M-1)$ matrix, and is said the dual matrix of $\operatorname{HOBW}(x)$

$D=\langle\operatorname{HOBW}(x), \operatorname{HOBW}(x)\rangle=\int_{0}^{1} \operatorname{HOBW}(x) H O B W^{T}(x) d x$

$H_{2}(z)=z \int_{0}^{z}\left(\frac{t^{k}}{z^{k}}\right) f\left(C^{T} \operatorname{HOBW}(t)\right) d t$

Now we collocate the Eq. (26) at the points $x_{i}=\frac{(2 i-1)}{2\left(\left(2^{k-1}\right)(M)\right)}$ yielding

$C^{T} \operatorname{HOBW}\left(x_{i}\right)=\alpha+\int_{0}^{x_{i}} H_{1}(z) d z-\int_{0}^{x_{i}} H_{2}(z) d z$,

To apply the Gaussian integration to Eq. (26), we first use the transformation $\tau=\frac{2}{x_{i}} z-1$

to transfer the interval of integration into the interval [ -1 , 1]. Now, Eq. (26) given as:

$C^{T} \operatorname{HOBW}\left(x_{i}\right)=\alpha+\frac{x_{i}}{2} \int_{-1}^{1} H_{1}\left(\frac{x_{i}}{2}(\tau+1) d \tau-\frac{x_{i}}{2} \int_{-1}^{1} H_{2}\left(\frac{x_{i}}{2}(\tau+1) d \tau\right.\right.$,

Using the Gaussian integration formula, we get

$$
\begin{aligned}
C^{T} \operatorname{HOBW}\left(x_{i}\right) \cong & \alpha+\frac{x_{i}}{2} \sum_{j_{1}=1}^{s_{1}} w_{j_{1}} H_{1}\left(\frac{x_{i}}{2}\left(\tau_{j 1}+1\right)\right) \\
& -\frac{x_{i}}{2} \sum_{j_{2}=1}^{s_{2}} w_{j_{2}} H_{2}\left(\frac{x_{i}}{2}\left(\tau_{j 2}+1\right)\right) .
\end{aligned}
$$

where $\tau_{j 1}$ and $\tau_{j 2}$ are $s_{1}$ and $s_{2}$ zeros of Legendre polynomials $p_{s_{1}+1}($.$) and p_{s_{2}+1}($.$) and w_{j_{1}}$ and $w_{j_{2}}$ are weights. The idea for the above approximation is the exactness of the Gaussian integration formula for polynomials of degree at most $2 s_{1}+1$ and $2 s_{2}+1$ Eq. (32) yields $\left(2^{k-1}\right)(M-1)$ nonlinear algebraic equations with the unknowns coefficient matrix C. By using Newton's method to solve this system, the values of $C$ are obtained and hence we get the solution.

\subsection{Convergence analysis}

In this section, an analysis of the speed of convergence for our numerical method will be obtained.

Theorem 1 The series solution $y_{m}(x)=$ $\sum_{i=1}^{k} \sum_{j=0}^{M} c_{i j} \operatorname{HOBW}_{i j}(x)=C^{T} \operatorname{HOBW}(x)$, where $C$ and $\operatorname{HOBB}(x)$ are defined in (6) converges to $y(x)$ then $\lim _{m \rightarrow \infty}\left\|y(x)-y_{m}(x)\right\|_{2}=0$.

Proof Let $L^{2}(\Re)$ is the Hilbert space, $y(x) \in L^{2}[0,1]$ is approximated by HOBW as Eq. (6):

Let $y(x) \cong \sum_{j=0}^{n} c_{i j} \operatorname{HOBW}_{i j}(x)$ for a fixed $i$ be the solution of the Eq. (2) where $\left.c_{i j}=D^{-1}\left\langle y(x), \operatorname{HOBW}_{i j}(x)\right\rangle\right)$ in which $\langle.,$.$\rangle denotes the inner product.$

Assume $\operatorname{HOBW}(x)=\operatorname{HOBW}\left(x_{m}\right)$ and assume $\delta_{m}=\left\langle y(x), \operatorname{HOBW}\left(x_{m}\right)\right\rangle$, the sequence of partial sum is $\left\{\sigma_{i}\right\}$ of $\left(\delta_{m} \mathrm{HOBW}\left(x_{m}\right)\right)$ Let $\left\{\sigma_{i}\right\}$ and $\left\{\sigma_{j}\right\}$ be the partial sums with $i \geq j$.

We show that $\left\{\sigma_{i}\right\}$ is a Cauchy sequence in Hilbert space. Let, $\sigma_{i}=\sum_{m=1}^{i} \delta_{m} \operatorname{HOBW}\left(x_{m}\right)$.

Now, 


$$
\begin{aligned}
\left\langle y(x), \sigma_{i}\right\rangle & =\left\langle y(x), \sum_{m=1}^{i} \delta_{m} \operatorname{HOBW}\left(x_{m}\right)\right\rangle \\
& =\sum_{m=1}^{i} \bar{\delta}_{m}\left\langle y(x), \operatorname{HOBW}\left(x_{m}\right)\right\rangle, \\
& =\sum_{m=1}^{i} \bar{\delta}_{m} \delta_{m} \\
& =\sum_{m=1}^{i}\left|\delta_{m}\right|^{2},
\end{aligned}
$$

so

$$
\begin{aligned}
\left\|\sigma_{i}-\sigma_{j}\right\|^{2} & =\left\|\sum_{m=1}^{i} \delta_{m} \operatorname{HOBW}\left(x_{m}\right)\right\|^{2} \\
& =\left\langle\sum_{m=j+1}^{i} \delta_{m} \operatorname{HOBW}\left(x_{m}\right), \sum_{m=j+1}^{i} \delta_{m} \operatorname{HOBW}\left(x_{m}\right)\right\rangle \\
& =\sum_{m=j+1}^{i} \sum_{m=j+1}^{i} \delta_{m} \bar{\delta}_{m}\left\langle\operatorname{HOBW}\left(x_{m}\right), \operatorname{HOBW}\left(x_{m}\right)\right\rangle \\
& =\sum_{m=j+1}^{i}\left|\delta_{m}\right|^{2}
\end{aligned}
$$

Hence,

$$
\left\|\sum_{m=j+1}^{i} \delta_{m} \operatorname{HOBW}\left(x_{m}\right)\right\|^{2}=\sum_{m=j+1}^{i}\left|\delta_{m}\right|^{2}, \quad i>j
$$

From Bessel's inequality as $i \rightarrow 0$ we have $\sum_{m=1}^{\infty}\left|\delta_{m}\right|^{2}$, convergent and hence

$\left\|\sum_{m=j+1}^{i} \delta_{m} \operatorname{HOBW}\left(x_{m}\right)\right\|^{2} \rightarrow 0$ as $i \rightarrow 0$.

Therefore,

$\left\|\sum_{m=j+1}^{i} \delta_{m} \operatorname{HOBW}\left(x_{m}\right)\right\| \rightarrow 0$,

and $\left\{\sigma_{i}\right\}$ is a Cauchy sequence and it converges to for example w (say). We assert that

$y(x)=w$.

Thus,

$\left\langle w-y(x), \operatorname{HOBW}\left(x_{m}\right)\right\rangle=\left\langle w, \operatorname{HOBW}\left(x_{m}\right)\right\rangle-\left\langle y(x), \operatorname{HOBW}\left(x_{m}\right)\right\rangle$.

Hence,

\section{SN Applied Sciences}

Table 1 Comparison of truncate solutions acquired by HOBW at $2^{k-1}=4$ and $M=3$ ADM for Example 1

\begin{tabular}{llllll}
\hline$x$ & HOBW & ADM & Exact & AE of HOBW & AE of ADM \\
\hline 0.1 & 0.011 & 0.011 & 0.011 & 0 & 0 \\
0.2 & 0.048 & 0.04800000004 & 0.048 & 0 & $4 \times 10^{-11}$ \\
0.3 & 0.117 & 0.1170000002 & 0.117 & 0 & $2 \times 10^{-10}$ \\
0.4 & 0.224 & 0.2240000003 & 0.224 & 0 & $3 \times 10^{-10}$ \\
0.5 & 0.375 & 0.3750000001 & 0.375 & 0 & $1 \times 10^{-10}$ \\
0.6 & 0.576 & 0.5760000004 & 0.576 & 0 & $4 \times 10^{-10}$ \\
0.7 & 0.833 & 0.83300000003 & 0.833 & 0 & 0 \\
0.8 & 1.152 & 1.1520000007 & 1.152 & 0 & $1 \times 10^{-9}$ \\
0.9 & 1.539 & 1.5390000009 & 1.539 & 0 & $9 \times 10^{-9}$ \\
\hline
\end{tabular}

Table 2 Maximum AE at several values of $2^{k-1}$ and $M$ for Example 2 via HOBW

\begin{tabular}{lllll}
\hline $2^{k-1}$ & $M$ & & & \\
\cline { 2 - 5 } & 7 & 11 & 15 & 19 \\
\hline 8 & $4.59 \times 10^{-8}$ & $8.02 \times 10^{-9}$ & $4.19 \times 10^{-10}$ & $7.38 \times 10^{-11}$ \\
12 & $5.47 \times 10^{-10}$ & $2.49 \times 10^{-12}$ & $3.17 \times 10^{-14}$ & $2.12 \times 10^{-15}$ \\
16 & $3.39 \times 10^{-11}$ & $2.95 \times 10^{-14}$ & $1.21 \times 10^{-15}$ & $4.24 \times 10^{-16}$ \\
\hline
\end{tabular}

$=\left\langle\lim _{i \rightarrow \infty} \sigma_{i}, \operatorname{HOBW}\left(x_{m}\right)\right\rangle-\delta_{m}$

$=\lim _{i \rightarrow \infty}\left\langle\sigma_{i}, \operatorname{HOBW}\left(x_{m}\right)\right\rangle-\delta_{m}$

$=\lim _{i \rightarrow \infty}\left\langle\delta_{m} \operatorname{HOBW}\left(x_{m}\right), \operatorname{HOBW}\left(x_{m}\right)\right\rangle-\delta_{m}$

$=\delta_{m}-\delta_{m}=0$.

The above relation possible only if $y(x)=\sigma$. so that $y(x)$ and $\sigma_{i}$ converges to the same value. It provides the convergence guarantee of the HOBW method.

\section{Illustrative numerical examples}

In this section, we will study the Lane-Emden with the initial value having a singularity at $x=0$, the high exactness of the solution acquired by HOBW method are demonstrated and then we compare all results with the required solution.

Example 1 Consider the singular differential equation of LEE [13].

$\left\{\begin{array}{l}y^{\prime \prime}(x)+\frac{2}{x} y^{\prime}(x)+y(x)=6+12 x+x^{2}+x^{3}, \quad 0 \leq x \leq 1 \\ y(0)=y^{\prime}(0)=0,\end{array}\right.$ 

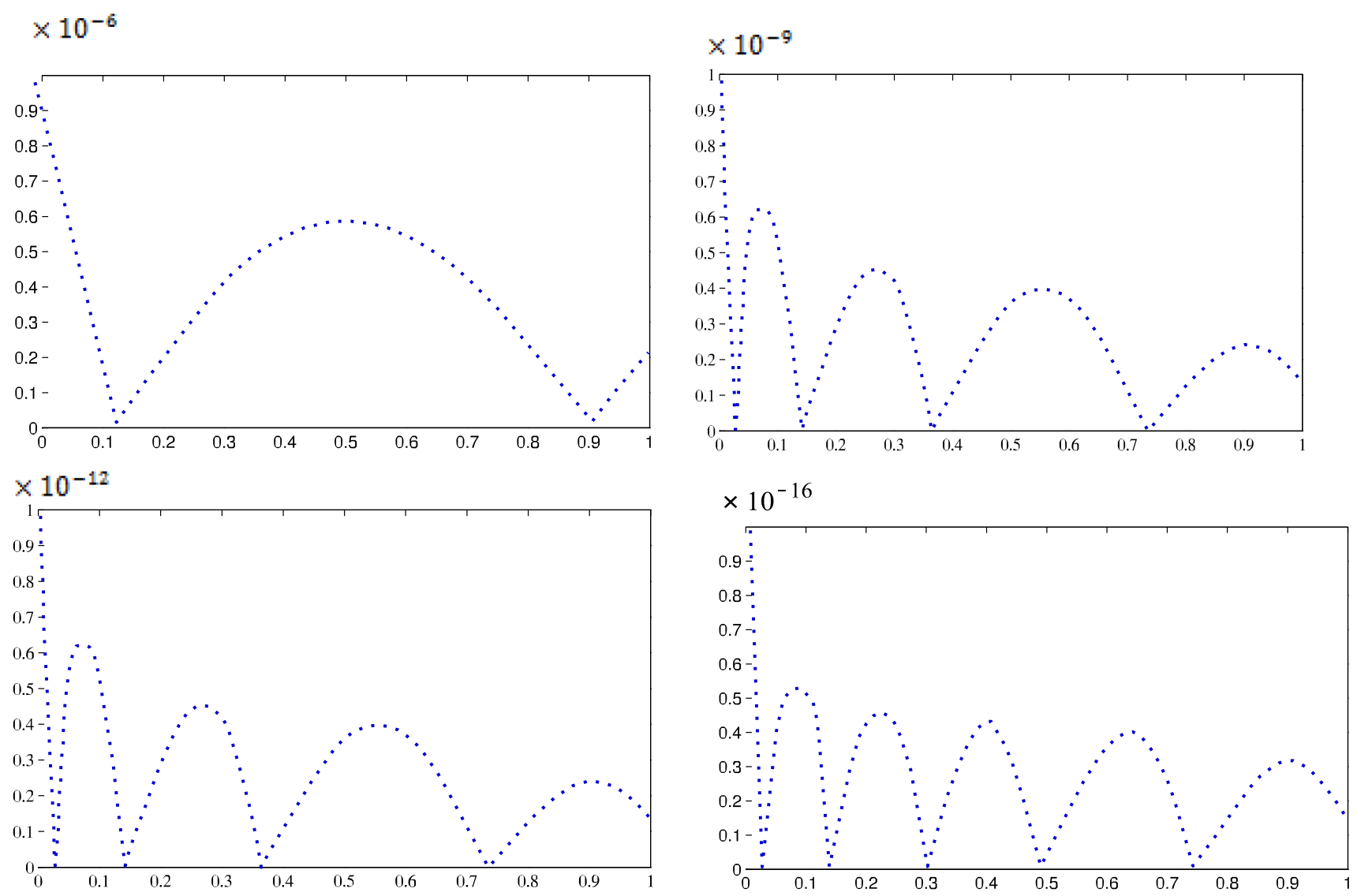

Fig. 1 Plot of the absolute error $(e(x))$ at $M=7,11,15$ and 19 for Example 2

with the correct solution $y(x)=x^{2}+x^{3}$.

Using the HOBW, the truncate solution $y(x)$ of (37) at $2^{k-1}=4$ and $M=3$ method, we get 16 number algebraic equations with the same number of unknowns and these equations are numerically solved by Newton's method with help of maple program, with the initial guess zero, we get the HOBW coefficients as: $\left\{\begin{array}{l}y^{\prime \prime}(x)+\frac{8}{x} y^{\prime}(x)+x y(x)=x^{5}-x^{4}+44 x^{2}-30 x, 0 \leq x \leq 1 \\ y(0)=y^{\prime}(0)=0\end{array}\right.$

with the correct solution $y(x)=x^{4}-x^{3}$

Table 2 compares the maximum AE of HOBW at several values of $2^{k-1}$ and $M$. These results have been included to

$C=[0,0,0.02083333333,0.07812500000,0.07812500000,0.1354166667,0.2291666667,0.3750000000$,

$0.3750000000,0.5208333333,0.7187500000,0.9843750000,0.9843750000,1.250000000,1.583333333,2]$

So, the truncate of $y(x)$ is $y(x)=C^{T} \operatorname{HOBW}(x)$ the acquired results have been compared with that of my seven order (ADM) [13] along with the required solutions and introduced in Table 1. The outcomes reveal that the results by HOBW, with using only a small number of bases, are very promising and superior to ADM and evaluated absolute errors (AE) by HOBW for $y(x)$ will be decreased rapidly in comparison with ADM.

Example 2 Consider the non-homogenous LEE [13]. demonstrate the validity and capability of HOBW (Fig. 1).

From Table 2, it is observed that our numerical method is more accurate. In order to show the accuracy of the proposed method, we have calculated the absolute error and the root means square error (RMS). These errors are given by

Absolute $\left(y\left(x_{i}\right)\right)=\left|y_{\text {exact }}\left(x_{i}\right)-y_{\text {approximation }}\left(x_{i}\right)\right|$, 
Table 3 Numerical solutions for Example 3 when $g=2, m=0$

\begin{tabular}{lllc}
\hline$x$ & Correct solution & HOBW solution & $\mathrm{AE}$ \\
\hline 0.1 & 0.9983333333 & 0.9983333334 & $1 \times 10^{-10}$ \\
0.2 & 0.9933333333 & 0.9933333321 & $1.2 \times 10^{-9}$ \\
0.3 & 0.9850000000 & 0.9850000002 & $1 \times 10^{-10}$ \\
0.4 & 0.9733333333 & 0.9733333364 & $3.1 \times 10^{-9}$ \\
0.5 & 0.9583333333 & 0.9583333336 & $1 \times 10^{-10}$ \\
0.6 & 0.9400000000 & 0.9400000015 & $1.5 \times 10^{-9}$ \\
0.7 & 0.9183333333 & 0.9183333331 & $1 \times 10^{-10}$ \\
0.8 & 0.8933333333 & 0.8933333344 & $1.1 \times 10^{-9}$ \\
0.9 & 0.8650000000 & 0.8650000056 & $5.6 \times 10^{-9}$ \\
\hline
\end{tabular}

Example 3 Consider the homogeneous form of the LEE [14].

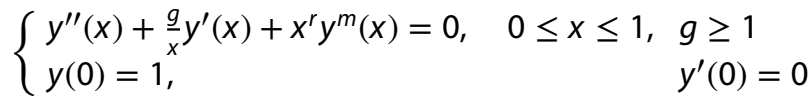

This equation transformed into VIDE form as follow

$$
y^{\prime}(x)=-\int_{0}^{x}\left(\frac{t^{g}}{x^{g}}\right) t^{r} y^{m}(t) d t, \quad y(0)=1, \quad g \geq 1
$$

We selected our example from [14], in which they solved the homogeneous form of LEE by ADM. Tables 3 and 4, exhibit the Numerical solutions of $y(t)$ by HOBW at $r=m=0 g=2$, and $r=0, m=1, g=2$.

a. For $r=m=0$, and $g=2$, the above equation has a correct solution

$y(x)=1-\frac{x^{2}}{6}$

Table 3 shows that, for $g=2, m=0$, the acquired results coincides with the required solution and efficiency of the method described through the AE.

By applying HOBW method, and taking $2^{k}=4, M=3$, HOBW coefficients $C$ is:

\section{$[1,1,0.9965277797,0.9795833334,0.9895833334,0.9826388889,0.97228222222$, $0.9583333334,0.9587333373,0.9444475445,0.9270874333,0.92625,0.90625$, \\ $0.8854166688,0.8617111111,0.8333333333]$.}

Table 4 A truncate solutions for Example 3 when $g=2, m=1$ b. For $r=0, m=1$, and $k=2$, Eq. (39) is:

$$
y^{\prime}(x)=-\int_{0}^{x}\left(\frac{t^{2}}{x^{2}}\right) y(t) d t, \quad y(0)=1
$$

\begin{tabular}{lllcl}
\hline$x$ & Correct solution & $\begin{array}{l}\text { HOBW solution } \\
\text { at } 2^{k}=4, M=3\end{array}$ & AE at $2^{k}=4, M=3$ & $\mathrm{AE} \mathrm{at} 2^{k}=8, M=7$ \\
\hline 0.1 & 0.9983341665 & 0.9983341428 & $2.37 \times 10^{-8}$ & $4.61 \times 10^{-14}$ \\
0.2 & 0.9933466540 & 0.9933467756 & $1.260 \times 10^{-7}$ & $7.02 \times 10^{-14}$ \\
0.3 & 0.9850673556 & 0.9850674729 & $1.173 \times 10^{-8}$ & $2.35 \times 10^{-14}$ \\
0.4 & 0.9735458558 & 0.9735458342 & $2.161 \times 10^{-8}$ & $5.11 \times 10^{-14}$ \\
0.5 & 0.9588510772 & 0.9588503494 & $7.278 \times 10^{-8}$ & $2.73 \times 10^{-13}$ \\
0.6 & 0.9410707892 & 0.9410707712 & $1.80 \times 10^{-8}$ & $6.35 \times 10^{-13}$ \\
0.7 & 0.9203109820 & 0.9203110906 & $1.086 \times 10^{-7}$ & $1.05 \times 10^{-11}$ \\
0.8 & 0.8966951136 & 0.8966952102 & $9.66 \times 10^{-8}$ & $3.24 \times 10^{-11}$ \\
0.9 & 0.8703632328 & 0.8703632216 & $1.12 \times 10^{-8}$ & $4.01 \times 10^{-11}$ \\
\hline
\end{tabular}


Table 5 A truncate and correct solutions for Example 4

\begin{tabular}{lllcr}
\hline $\mathrm{x}$ & Correct solution & HOBW at $2^{k}=16, M=15$ & $\begin{array}{l}\text { AE of HOBW at } \\
2^{k}=16, M=15\end{array}$ & AE of SDMM \\
\hline 0.25 & 1.06449445891786 & 1.06449445891789 & $3 \times 10^{-15}$ & $1.77 \times 10^{-13}$ \\
0.5 & 1.28402541668774 & 1.28402541668779 & $5 \times 10^{-15}$ & $2.14 \times 10^{-13}$ \\
0.75 & 1.75505465696030 & 1.75505465696073 & $4.3 \times 10^{-14}$ & $2.93 \times 10^{-13}$ \\
1 & 2.71828182845904 & 2.71828182845916 & $1.2 \times 10^{-14}$ & $4.54 \times 10^{-13}$ \\
\hline
\end{tabular}

The correct solutions for this problem are given by $y(x)=\frac{\sin (x)}{x}$ applying HOBW method, and taking $2^{k}=4, M=3$, we note that HOBW coefficients $C$ as
For examples 4, we have very less $A E$ by increasing the order of HOBW.

$[0.9999991692,1.000102884,0.9961198453,0.9896150092,0.9896150391,0.9827174717$,

$0.9723985577,0.9588502916,0.9518504492,0.9453088641,0.928552389035$,

$0.9088509723,0.9085510555,0.8891691113,0.8165704733,0.8414703828]$

applying this method and taking $2^{k}=8, M=7$, if $2^{k}, M$ increases, the truncate solution gets the required solution. The truncate solutions acquired by HOBW for shape factor $g=2$ and $2^{k}=4, M=3$, at $2^{k}=8, M=7$ with require solutions and AE demonstrated in Table 2. By increasing, $2^{k}, M$ the computed results have appropriated the exactness was increased as well. and the truncate solution gets an approximate to the correct solution as demonstrated in Table 4. The CPU time for running a case may depend on the choice of $2^{k}$, Mfor solving the system of linear algebraic equations resulting from the discretized equations. If a different value $2^{k}, M$ would have been used, the CPU time would be different.

Example 4 Consider the LEE type which is of second order homogeneous singular several equations given in [14].

$\left\{\begin{array}{l}y^{\prime \prime}(x)+\frac{2}{x} y^{\prime}(x)-2\left(2 x^{2}+3\right) y(x)=0,0 \leq x \leq 1 \\ (0)=1, y^{\prime}(0)=0\end{array}\right.$

with the correct solution $y(x)=e^{x^{2}}$.

In Table 5 a comparison between these results with the second derivative multistep method (SDMM) in [14] is also given. The results acquired by HOBW, by using $2^{k}=16, M=15$ are very promising and superior to those of SDMM. It is demonstrated that, for a certain value of $M$, as $2^{k}$ increases, the exactness increases and for a certain value of $2^{k}$, as $M$ increases, the exactness increases as well. Therefore, HOBW for solving this problem is very effective and more accurate as compared with the second derivative multistep method.

The results acquired by the present method are compared with the correct solution as demonstrated in Table 5.

\section{Conclusion}

In this paper, we introduced VIDE as equivalent to the LEE. The acquired VIDE form of LEE to overcome the difficulty of the singular behavior at $x=0$. The HOBW has been connected for settling the most prominent differential conditions. Despite this reality, such a model gives an imperative hypothetical point of view concerning the hypothesis, as one may see this as the fringe between polytropic one that is physically attainable. It is additionally important to take note of that such an outstanding model has, despite the unending range, a limited mass. Moreover, other outstanding models, which are made in a "layered" form where each layer comprises of a polytope of an alternate record, may likewise use this capacity for a bit of the star, in which case a limited sweep would be conceivable. Notwithstanding these relations, there are likewise a few different determinations that one can make from the polytropic model of stars. For relations of this sort, there exists a connection between the polytropic file, the mass of a star, and the span. It is maybe apparent in the talk of the investigative arrangements of the polytropic record that one could gather a connection between the polytropic file of the star and the range that one would ascertain from that star. The class of the equations was released, by changing the coefficient $y^{\prime}$ and the method was introduced. Using this method, the VIDE forms is reduced to algebraic equations. We got these equations involving $2^{k} \times(M-1)$ variables which solved using Newton's iterative method through MAPLE program. It was evident that the method for a certain value of $M$ as $2^{k}$ increased, the exactness was increased, and also for a certain value of $M$, as $M$ increased, the exactness was increased high as well. 
Acknowledgements The authors are grateful to the anonymous referee for his suggestions, which have greatly improved the presentation of the paper.

Authors' contributions The author has made an equal contribution. The author read and approved the final manuscript.

Funding This research work is not supported by any funding agencies.

\section{Compliance with ethical standards}

Competing interests The authors declare that they have no competing interests.

\section{References}

1. Kaur H, Mittal RC, Mishra V (2013) Haar wavelet approximate solutions for the generalized Lane-Emden equations arising in astrophysics. Comput Phys Commun 184(9):2169-2177

2. Luo T, Xin Z, Zeng $H$ (2016) On nonlinear asymptotic stability of the Lane-Emden solutions for the viscous gaseous star problem. Adv Math 291(1):90-182

3. Deniz Sinan, Bildik Necdet (2017) A new analytical technique for solving Lane-Emden type equations arising in astrophysics. Bull Belgian Math Soc-Simon Stevin 24(2):305-320

4. Bildik Necdet, Deniz Sinan (2018) New analytic approximate solutions to the generalized regularized long wave equations. Bull Korean Math Soc 55(3):749-762

5. Bildik Necdet, Deniz Sinan (2018) Solving the Burgers' and regularized long wave equations using the new perturbation iteration technique. Numer Methods Partial Differ Equ 34(5):1489-1501

6. Wazwaz AM, Rach R, Duan J-S (2013) Adomian decomposition method for solving the Volterra integral form of the LaneEmden equations with initial values and boundary conditions. Appl Math Comput 219(10):5004-5019

7. Aslanov A (2016) An elegant correct solution for the EmdenFowler equations of the first kind. Math Methods Appl Sci 39(5):1039-1042

8. Wazwaz AM (2005) Analytical solution for the time-dependent Emden-Fowler type of equations by Adomian decomposition method. Appl Math Comput 166(3):638-651

9. Bencheikh A, Chiter L, Abbassi H (2017) Bernstein polynomials method for numerical solutions of integro-severalial form of the singular Emden-Fowler initial value problems. J Math Comput Sci 17(1):66-75
10. Boubaker K, Van Gorder RA (2012) Application of the BPES to Lane-Emden equations governing polytropic and isothermal gas spheres. New Astron 17:565-569

11. Aslanov A (2009) Approximate solutions of Emden-Fowler type equations. Int J Comput Math 86:807-826

12. Harley C, Momoniat E (2008) Instability of invariant boundary conditions of a generalized Lane-Emden equation of the second-kind. Appl Math Comput 198:621-633

13. Ramadan MA, Ali MR (2017) Numerical solution of Volterra-Fredholm integral equations using hybrid orthonormal bernstein and block-pulse functions. Asian Res J Math 4(4):1-14

14. Ramadan MA, Ali MR (2017) Application of Bernoulli wavelet method for numerical solution of fuzzy linear Volterra-Fredholm integral equations. Commun Math Model Appl 2(3):40-49

15. Ramadan MA, Ali MR (2017) Solution of integral and integro-differential equations system using hybrid orthonormal bernstein and block-pulse functions. J Abstract Comput Math 2(1):35-48

16. Parand K, Khaleqi S (2016) The rational Chebyshev of second kind collocation method for solving a class of astrophysics problems. Eur Phys J Plus 131:1-24

17. Sadat R, Kassem M (2018) Explicit solutions for the (2+1)-dimensional Jaulent-Miodek equation using the integrating factors method in an unbounded domain. Math Comput Appl 23(1):1-9

18. Ramadan MA, Ali MR (2017) An efficient hybrid method for solving fredholm integral equations using triangular functions. New Trends Math Sci 5(1):213-224

19. Ali MR, Hadhoud AR (2019) Hybrid orthonormal Bernstein and Block-Pulse functions wavelet scheme for solving the 2D Bratu problem. Results Phys 12:525-530

20. Hojjati G, Parand K (2011) An efficient computational algorithm for solving the nonlinear Lane-Emden type equations. World Acad Sci Eng Technol 5:182-187

21. Ali MR (2018) Darboux transformation for soliton solutions of the modified Kadomtsev-Petviashvili-II equation. Commun Math Model Appl 3(3):28-36

22. Parand K, Roozbahani Z, Bayat-Babolghani F (2013) Solving nonlinear Lane-Emden type equations with unsupervised combined artificial neural networks. Int J Ind Math 5(4):355-366

23. Parand K, Pirkhedri A (2010) Sinc-collocation method for solving astrophysics equations. New Astron 15(6):533-537

24. Hossayni SA, Rad JA, Parand K, Abbasbandy S (2015) Application of the exact operational matrices for solving the Emden-Fowler equations, arising in astrophysics. Int J Ind Math 7(4):351-374

25. Parand K, Hemami M (2016) Numerical study of astrophysics equations by Meshless collocation method based on compactly supported radial basis function. Int J Appl Comput Math 3(2):1053-1075

26. Ali MR (2018) Solution of KdV and boussinesq using Darboux transformation. Commun Math Model Appl 3(3):16-27 